\title{
Évaluation et communication des nouvelles organisations d'interface : le cas des réseaux de santé
}

\section{Christian Bourret}

\section{OpenEdition} Journals

Édition électronique

URL : http://journals.openedition.org/communicationorganisation/1411

DOI : 10.4000/communicationorganisation. 1411

ISSN : 1775-3546

\section{Éditeur}

Presses universitaires de Bordeaux

\section{Édition imprimée}

Date de publication : 1 décembre 2010

Pagination : 77-88

ISBN : 978-2-86781-743-4

ISSN : $1168-5549$

\section{Référence électronique}

Christian Bourret, «Évaluation et communication des nouvelles organisations d'interface : le cas des réseaux de santé ", Communication et organisation [En ligne], 38 | 2010, mis en ligne le 01 décembre 2013, consulté le 04 mai 2019. URL : http://journals.openedition.org/

communicationorganisation/1411; DOI : 10.4000/communicationorganisation. 1411 


\title{
Évaluation et communication des nouvelles organisations d'interface: le cas des réseaux de santé
}

\author{
Christion BOURRET ${ }^{01}$
}

\section{Introduction}

Le poids financier des systèmes de santé dans les économies nationales s'est accentué dans tous les pays développés. En France, il approche désormais les $12 \%$ du PIB, le budget social de la Nation dans son ensemble étant devenu supérieur à celui de l'État. Le vote de la loi HPST (Hôpital, Patients, Santé, Territoires), et notamment la création des ARS (Agences Régionales de Santé), effective en avril 2010, symbolise les changements en cours, avec la volonté des pouvoirs publics de promouvoir une nouvelle gestion territorialisée de la santé, dans un contexte de ressources limitées.

Il s'agit en particulier d'essayer de faire coopérer, en dépassant les cloisonnements, notamment entre médecine de ville et secteur hospitalier, mais aussi entre professions, et donc d'essayer de mieux concilier différentes logiques. Dans cette perspective, depuis une trentaine d'années, la France cherche à développer des organisations d'interface, en particulier les réseaux de santé. Leur expérimentation a rencontré la question de l'ambivalence de leur évaluation dans une perspective de démarche qualité: aide au développement et amélioration mais aussi contrôle (voire sanction).

Nous évoquerons tout d'abord le contexte de l'affirmation de cette nouvelle forme d'organisation d'interface en santé, constituant une expérimentation innovante avec de forts enjeux informationnels et communicationnels. Nous analyserons ensuite la rencontre de l'évaluation et des réseaux de santé. Nous proposerons enfin une approche SIC (Sciences de l'Information et de la Communication) de l'évaluation des réseaux de santé, pouvant être étendue à d'autres organisations d'interface en santé.

01. Christian Bourret est Maître de Conférences en Sciences de l'Information et de la Communication HDR à I'Université Paris Est Marne-la-Vallée. II est chercheur au sein de l'équipe DICEN (Dispositifs d'Information et de Communication à l'Ere Numérique) du CNAM (Conservatoire National des Arts et Métiers), reconnue également par l'Université Paris Est Marne-la-Vallée. Mail : bourret@univ-mlv.fr 


\section{L'affirmation d'une organisation d'interface en santé pour dépasser les cloisonnements}

Comme le projet ou l'innovation, le réseau est une notion clé de la société post-industrielle ou d'hypermodernité. Dans cette société d'« individualisme connecté ", le besoin de "reliance » est important. C'est particulièrement le cas en France. La traditionnelle faiblesse des organisations d'interface y correspond à un choix de la Révolution française de supprimer les corps intermédiaires et, notamment les corporations, considérées comme des symboles de l'Ancien Régime. Il a fallu attendre 1884 pour que soient reconnus les syndicats et 1901 les associations.

Le système de santé français se caractérise par de nombreux cloisonnements entre secteurs d'activité s'appuyant sur des logiques et des modalités de gestion différentes. Le rôle du ministère de la Santé (réglementation, pilotage du système de santé, financement par l'impôt) est distinct de celui de l'Assurance Maladie (financement par les cotisations sociales). Le système de santé français repose sur deux secteurs à logiques différentes: celui des soins primaires de la médecine de ville, dit « libéral », et à approche plutôt individualiste (privée) et le secteur hospitalier ( $45 \%$ des dépenses), en y distinguant secteur public et secteur privé (cliniques). Les clivages sont aussi nets entre professions, notamment entre le diagnostic (médecins) et les soins (infirmières) et, également, entre le médical et le social.

À partir de 1984, l'épidémie du Sida a bousculé ces clivages, amenant à chercher de nouvelles coopérations entre médecine de ville et secteur hospitalier. Des médecins de ville ont été à l'initiative de réseaux de santé «spontanés» ou «militants», développant une approche globale de la santé au sens de l'OMS (Organisation Mondiale de la Santé), articulée à une dimension sociale (lutte contre la précarité) et plus seulement limitée aux soins. Dans le même temps, les patients, et, en particulier les malades atteints du Sida, sont devenus davantage acteurs de leur santé. Ces réseaux «spontanés » ont pu s'opposer à des réseaux dits «coordonnés de soins ", correspondant à des initiatives de l'Assurance Maladie, de la Mutualité Sociale Agricole ou d'assureurs (Groupama...) pour promouvoir de nouvelles modalités de rémunération (différentes du paiement à l'acte habituel dans la médecine libérale) et une certaine maîtrise des dépenses sur le modèle des HMOs (Health Maintenance Organizations) américaines. Les ordonnances d'avril 1996 ont valorisé les réseaux de soins coordonnés. La loi du 4 mars 2002 relative aux Droits des malades et à la qualité du système de santéa essayé de concilier cette approche avec celle de réseaux de santé émanant de médecins de terrain dans des zones difficiles, en donnant une définition de synthèse ${ }^{02}$.

02. Les réseaux de santé ont pour objet de favoriser l'accès aux soins, la coordination, la continuité ou l'interdisciplinarité des prises en charge sanitaires, notamment de celles qui sont spécifiques à certaines populations, pathologies ou activités sanitaires. Ils assurent une prise en charge adaptée aux besoins de la personne, tant sur le plan de l'éducation à la santé, de la prévention, du diagnostic, que des soins. Ils 
Elle a également insisté sur la notion de « démocratie sanitaire ", mettant à la fois l'accent sur le rôle et la responsabilisation de tous les acteurs, y compris patients, processus qui est encore largement à construire.

Les réseaux de santé qui se sont développés depuis le début des années 1980 sont au nombre d'environ 700, regroupant entre 150000 et 200000 patients. Une brochure de la Haute Autorité de Santé (HAS) de 2006 les présente comme "une démarche d'amélioration de la qualité ». Ils constituent une expérimentation correspondant à une « innovation ordinaire » ou au quotidien (au sens de N. Alter ${ }^{03}$ [ALTER, 2005]): par leur positionnement d'interface entre la médecine de ville et le secteur de l'hospitalisation, par l'intégration de dispositifs socio-techniques (dossiers informatisés patients et systèmes d'information) pour promouvoir de nouvelles pratiques coopératives, avec toute l'importance de la négociation. Ils correspondent aussi à de nouvelles formes de partenariats public/privé, notamment entre médecine de ville privée et secteur hospitalier public. Ils constituent également des espaces de reconnaissance non hiérarchiques pour des médecins libéraux qui se sentaient souvent «méprisés » par l'hôpital. Cela a été aussi le cas pour d'autres professions qui y ont vu leur rôle propre davantage reconnu : kinésithérapeutes, par exemple dans les réseaux bronchiolite du nourrisson, sages-femmes dans les réseaux de périnatalité, ou infirmiers et infirmières, qui ont pu devenir responsables ou coordonnateurs de réseaux de santé.

Espaces projets innovants ou formes organisationnelles « dissipatives » [LE MOENNE, 2004], les réseaux de santé constituent un terrain de recherche privilégié pour les SIC, en articulant comme le propose F. Bernard ${ }^{04}$ les problématiques du lien, du sens, du savoir et de l'action. Les réseaux de santé sont aussi des « organisations entre projets et récits », avec toute l'importance des actes de langage ${ }^{05}$, la communication permettant également d'analyser le changement ${ }^{06}$.

L'enjeu majeur des réseaux de santé est de dépasser des logiques individuelles ou d'institutions pour proposer des approches davantage coopératives. La coordination des activités y est essentielle pour passer de pratiques individuelles à des pratiques coopératives construites autour de chaque patient. La coopération se construit progressivement par les interactions entre les différents partenaires et l'émergence de nouveaux savoirs partagés (y compris des patients). Toujours dans une approche relevant des SIC, les réseaux de

peuvent participer à des actions de santé publique. Ils procèdent à des actions d'évaluation afin de garantir la qualité de leurs services et prestations".

03. ALTER N., L'innovation ordinaire, Paris, PUF, 2000, rééd. Coll. Quadrige, 2005, 288 p

04. BERNARD F. Les SIC une discipline de l'ouverture et du décloisonnement. In: BOUZON A. dir. La communication organisationnelle en débat. Champs, concepts, perspectives. Paris: L'Harmattan, 2006, p. 33 - 46.

05. GRAMACCIA G., Les actes de langage dans les organisations, Paris, L'Harmattan, 2001, $287 \mathrm{p}$.

06. CARAYOL V. Communication organisationnelle. Une perspective allagmatique. Paris: L'Harmattan, 2004, $235 \mathrm{p}$. 
santé peuvent constituer des espaces projets pour favoriser le développement de la «démocratie sanitaire »: construction de la confiance entre tous les partenaires, en s'appuyant en particulier sur des outils relevant des TIC (technologies de l'information et de la communication) pour modifier, dans le sens des patients, la traditionnelle asymétrie d'information en faveur des médecins.

Les réseaux de santé peuvent être ainsi abordés en termes de situations d'information et de communication, dans une approche systémique, en particulier à travers la grille de lecture de la sémiotique situationnelle proposée par A. Mucchielli [MUCCHIELLI, 2010], qui décompose la situation pour un acteur en différents cadres ou contextes d'interprétation: positionnement des acteurs, enjeux, contexte spatio-temporel, normes, valeurs, qualité des relations.

Toute expérimentation se traduisant par un financement contractuel dans une logique objectifs/résultats, la question de l'évaluation des réseaux de santé s'est très vite posée.

\section{La rencontre de l'évaluation et des réseaux de santé}

L'évaluation est consubstantielle aux approches qualité et à la notion de normes ${ }^{07}$. Pour l'Afnor (Association Française de Normalisation), les normes sont un outil d'amélioration de la qualité, en tenant compte du contexte. L'importance prise par les normes a été analysée, et notamment les tensions possibles entre normes organisationnelles et professionnelles [BOUSSARD, 2008]. L. Thévenot [THEVENOT, 1997] avait déjà pointé l'évolution vers un "gouvernement par les normes ", liée à l'essor du marché et notamment à la construction du marché européen, avec l'extension de la normalisation de la production des biens à celle des compétences des personnes (avec le risque d'uniformisation de l'information). Pour C. Le Moënne, il s'agit « sans doute du mouvement de normalisation managériale le plus vaste, le plus systématique et le plus radical depuis l'origine du capitalisme » [LE MOËNNE, 2004]. L'évaluation a commencé à se développer dans les pays anglo-saxons et dans les entreprises privées. En France, elle s'est progressivement étendue au secteur public à partir des années 1980, le NMP (Nouveau Management Public) prenant la suite du PPBS (Program Planning Budgeting System) et de la RCB (Rationalisation des Choix Budgétaires). Lidée principale du NMP est de transposer les méthodes de gestion du secteur privé au secteur public pour en améliorer l'efficience. En France, depuis 1996, chaque année, le Parlement vote la LFSS (Loi de Financement de la Sécurité Sociale).

07. Selon l'Afnor, une norme est un « document, établi par consensus et approuvé par un organisme reconnu, qui fournit, pour des usages communs et répétés, des règles, des lignes directrices ou des caractéristiques, pour des activités ou leurs résultats, garantissant un degré optimal d'ordre dans un contexte donné » (définition émanant de I'ISO - International Standards Organization - et reprise au niveau européen). 
La logique objectifs/résultats imposée par la LOLF (Loi Organique des Finances Publiques) modifie progressivement les modalités de gestion du secteur public, y compris des universités (tableaux de bord, indicateurs). Le secteur de la santé a été concerné par cette évolution.

Devenue progressivement incontournable depuis la fin des années 1980, l'évaluation a été aussi contestée, notamment dans son obsession de l'efficience, perçue avant tout dans une approche quantitative et de stricte application de normes, oubliant notamment l'analyse de la spécificité du contexte de leur mise en place. Les analyses de C. Dejours [DEJOURS, 2010] sur les dérives d'une évaluation des performances et des compétences trop individualisée pouvant correspondre à de nouvelles formes de souffrance au travail rejoignent les critiques de V. De Gaulejac [DE GAULEJAC, 2005] sur la « société malade de la gestion » et celles d'H. Mintzberg [MINTZBERG, 2001] (« remarque sur un bien vilain mot: efficience »).

L'approche qualité ne peut pas se réduire à la stricte application de normes et devenir uniquement une prescription et un contrôle. Elle est avant tout une culture, un état d'esprit, un comportement d'amélioration et d'adaptation aux situations et aux contextes. L'évaluation des réseaux de santé doit ainsi constituer un état d'esprit et susciter l'adhésion de tous les acteurs [SCHWEYER, 2002]

En France, quand elle se conjugue avec la faiblesse de la culture du partage de l'information, la difficulté à mettre en place les procédures d'évaluation peut être jugée comme un facteur négatif, en particulier dans le secteur de la santé. L'évaluation repose sur la fiabilité des données. Or, produire des données pour soi-même, n'est pas la même chose que d'en produire pour d'autres, surtout quand ces données peuvent servir à un contrôle ou à une évaluation.

Les réseaux de santé ont très vite été évalués. L'évaluation « s'inscrit dans les règles de fonctionnement du réseau " [SCHWEYER, 2002]. Elle en constitue même un "principe constitutif », étant indissociable de la charte du réseau et de sa convention constitutive. Le rapport d'évaluation triennal constitue « le point d'orgue de la démarche d'évaluation ». La démarche d'évaluation comporte trois temps: d'anticipation, de suivi et de réaction (ajustement) [BARRÉ, 2005]. L'évaluation triennale d'un réseau gérontologique nous a permis d'analyser ces enjeux. La difficulté est d'évaluer des activités différentes (médicales, administratives...), à la fois individuelles (médecins libéraux) et collectives (hôpitaux), s'appuyant sur des outils relevant des TIC (systèmes d'information, dossiers patients partagés...).

J. Plante, convoqué par P. Lievre, définit « l'évaluation comme la formation d'un jugement de valeur sur une action, dans une perspective de prise de décision ». Pour lui, « la qualité d'une évaluation tient au degré d'intégration des résultats produits dans les représentations à venir des commanditaires » [LIEVRE, 2002]. Les premiers réseaux de soins coordonnés y ont consacré 
des sommes importantes, avec parfois des dérives en évaluation - sanction. À partir de la loi de mars 2002 qui a consacré les réseaux de santé, s'est affirmée, dans une perspective de gestion de projet et de démarche qualité, une évaluation - amélioration comme aide à leur développement. Elle a associé évaluation interne (avec une dimension formation) et évaluation externe, confiée à des organismes extérieurs (le plus souvent cabinets de consultants). Une évolution s'est produite progressivement avec le passage d'une obligation de moyens à celle de résultats avec également l'évaluation de la satisfaction des usagers. Dans un réseau, les professionnels de santé peuvent être à la fois producteurs et consommateurs de ressources. Évaluer, c'est apprécier à la fois la qualité de services offert aux professionnels et la prestation fournie par les professionnels impliqués dans le réseau. C'est aussi juger le fonctionnement du réseau, la qualité de prise en charge des patients et son influence sur son territoire [BARRÉ, 2005].

En faisant référence à E. Monnier, P. Lievre a insisté sur ce qui différencie l'évaluation de l'audit et du contrôle: la construction du système de référence avec l'implication des acteurs qui vont construire la grille de lecture utilisée pour juger leur action [LIEVRE, 2002]. L'évaluation met en relation objectifs, résultats et moyens. L'efficacité correspond à l'analyse des résultats en regard des objectifs fixés. Elle mesure l'atteinte des objectifs. L'efficience est le rapport entre les résultats et les objectifs en tenant compte des moyens utilisés. Il s'agit de se situer à l'interface d'une logique du souhaitable (objectifs) et d'une logique du possible, en intégrant les contraintes du terrain (contexte), les moyens consacrés à l'action et son caractère innovant [LIEVRE, 2002].

Un tournant important a été pris en 2006 avec le rapport de l'IGAS (Inspection Générale des Affaires Sociales) sur le financement des réseaux de santé ${ }^{08}$. En mettant néanmoins en évidence certains succès et en conseillant de poursuivre leur expérimentation, ce rapport controversé a considéré les " réseaux de santé (comme) plus que décevants ». Il a insisté sur les impératifs d'évaluation, de nécessaire lisibilité de l'activité des réseaux de santé et de service rendu. Ce rapport a correspondu à une diminution du financement des réseaux de santé. Certains responsables de réseaux de santé font le lien avec la nécessité de trouver des financements pour d'autres projets, comme par exemple celui de DMP (Dossier Médical Personnel), jugé alors prioritaire.

Dans le prolongement du rapport de l'IGAS, une circulaire de mars $2007^{\circ 9}$ sur l'évolution des réseaux de santé a été largement consacrée à leur évaluation. Elle précise tout d'abord les objectifs des réseaux de santé: "assurer la cohérence des parcours de soins et répondre à une logique d'organisation

08. DANIEL C., DELPAL B., LANNELONGUE C., Contrôle et évaluation du fonds d'aide à la qualité des soins de ville (FAQSV) et de la dotation de développement des réseaux (DDR), rapport Inspection Générale des Affaires Sociales (IGAS), Paris, La Documentation française, 2006, 163 p.

09. Circulaire conjointe de la Caisse Nationale d'Assurance Maladie et de la Direction de l'Hospitalisation et de l'Organisation des Soins (DHOS) du Ministère de la Santé du 2 mars 2007 
territoriale en permettant une prise en charge globale des patients et le décloisonnement des professionnels ». Le texte donne ensuite sa définition de l'évaluation: «L'évaluation des réseaux s'entend comme l'appréciation de l'atteinte d'objectifs qualitatifs et quantitatifs permettant de mesurer l'impact des réseaux sur la qualité de la prise en charge des patients, les pratiques des professionnels de santé et l'environnement sanitaire du réseau ». La mise en place d'un processus d'évaluation est devenue obligatoire pour toute demande de financement et ses modalités (internes et externes) sont bien définies.

La difficulté est d'évaluer une organisation innovante, expérimentale, fortement contextualisée, en évolution permanente et de bien préciser les objectifs de l'évaluation et son contexte, avec l'incertitude des comparaisons (chaque réseau étant différent). Comme l'avait souligné B. Kouchner, alors ministre de la Santé, lors des journées nationales des réseaux de santé en 2001, le défi est de «formaliser sans stériliser ». Ce défi est celui de l'ambivalence de l'évaluation déjà évoquée, à la fois outil d'aide au changement et de contrôle. En outre, dans un réseau de santé, les situations des acteurs peuvent changer: producteurs ou utilisateurs de ressources. De plus en plus évalués et contrôlés, les réseaux de santé sont aussi désormais en concurrence avec d'autres organisations d'interface (notamment maisons de santé pluriprofessions qui semblent avoir la faveur des pouvoirs publics et structures d'HAD: hospitalisation à domicile).

Pour C. Dejours, «l'essentiel du travail ne se voit pas et ne s'observe pas ", toute cette "partie immergée de l'iceberg, échappe à l'évaluation, aussi bien qualitative que quantitative " [DEJOURS, 2010], c'est notamment la différence entre le travail effectif et le travail prescrit. Il rejoint ainsi D. Bougnoux, qui, en insistant sur le "primat de la relation » pour les sciences de la communication, avait lui aussi souligné que « le monde des relations est par définition peu visible » [BOUGNOUX, 2001].

C'est dans ce contexte en pleine évolution et en étant conscient des difficultés de la démarche, que nous avons participé, dans le cadre d'une recherche-intervention, à l'évaluation de réseaux de santé, en particulier d'un réseau gérontologique d'une grande ville de province.

\section{Pour une approche SIC de l'évaluation des réseaux de santé}

Notre implication dans l'évaluation de réseaux de santé s'intègre dans un positionnement épistémologique relevant à la fois du constructivisme social dans une approche systémique et de "l'intelligence de la complexité » [MORIN'03], en s'appuyant notamment surla grille delecture des « dialogies » : rechercher des complémentarités entre des notions habituellement considérées comme opposées, comme, par exemple, l'ordre et le désordre.

Confronté à la diversité des représentations de l'évaluation émanant des différents acteurs des réseaux de santé évalués, nous avons essayé de nous placer dans la position de « neutralité engagée » définie par N. Heinich : « la neutralité 
est souvent la seule ressource pour comprendre la logique des uns et des autres, et parfois la faire comprendre aux uns et aux autres » [HEINICH'06]. Nous nous situons aussi dans la perspective tracée par C. Dejours, quand il souligne la différence entre juger et mesurer, "l'ambiguïté de la notion d'évaluation (étant) dans l'écart entre jugement et mesure ». Pour lui, « travailler, c'est avant tout vivre ensemble». Il propose de « repenser l'évaluation » pour aller vers une évaluation "équitable ", insistant sur la notion de reconnaissance, pour analyser dans la coopération ${ }^{10}$ les ressorts de la motivation et de l'adhésion. Cette évaluation «équitable » donne toute sa place au chercheur: elle repose sur le couplage permanent avec des recherches de terrain sur le travail concret et effectif. « Evaluer, c'est savoir écouter la parole au travail » pour avoir accès à la partie non visible de l'iceberg de l'activité [DEJOURS, 2010]. De leur côté, $V$. de Gaulejac et al. ont proposé le concept d' "évaluation dynamique », « mobilisant toutes les capacités réflexives et créatrices » [DE GAULEJAC, 2005].

Les réseaux de santé se situent entre récit et projet [D'ALMEIDA, 2006]. Correspondant à un espace projet, ils se construisent par les récits de leurs membres, les actes de langage contribuant au développement du «lien communicationnel » [GRAMACCIA, 2001]. Le processus d'évaluation est à la fois un projet ou une démarche qualité s'appuyant sur des récits, traduisant des approches et des représentations différentes de l'évaluation. Dans le réseau étudié, aux discours favorables et assez généraux des responsables ou des représentants des institutions le finançant, s'opposaient ceux des personnels de terrain (cellule de coordination), qui voyaient principalement dans l'évaluation à la fois une perte de temps et un risque d'obligation de contrôle de l'activité des partenaires qu'ils ne voulaient pas assumer. Au cours de notre recherche, notre approche de l'évaluation a évolué. Il nous a fallu nous faire accepter. Pour nous, le rôle de chercheur-évaluateur relève désormais davantage d'une "maïeutique ", d'une posture de facilitateur, de « traducteur ». Il nous a fallu convaincre, donner envie de travailler avec nous, puis aider à préciser les idées, essayer de formuler des représentations, tout cela pour construire un sens partagé entre tous les membres du réseau, les évaluateurs et les tutelles qui financent. Notre approche repose davantage sur les interactions avec les acteurs de terrain et sur la recherche de convergence des représentations entre chercheurs et équipe de coordination. Dans cette perspective, comme l'a envisagé P. Lievre [LIEVRE'02], l'évaluation correspond bien à "un processus d'apprentissage collectif » avec une forte dimension d'écoute et pédagogique d'aide au développement (formation).

L'approche de l'évaluation-amélioration que nous proposons correspond à celle envisagée par F. Bernard [BERNARD'06] pour les SIC: donner du sens à l'action pour créer du lien en s'appuyant sur des savoirs de plus en plus

10. La coopération correspond alors à une volonté, une envie de travailler ensemble, la coordination davantage à une prescription reposant sur des procédures. 
partagés et collectifs. Il s'agit d'intégrer progressivement les représentations de tous les acteurs pour essayer de construire des connaissances partagées, voire une intelligence collective.

Nous retrouvons ainsi à la fois H. Mintzberg [MINTZBERG, 2001] et V. de Gaulejac qui propose de « redonner du sens à l'action », en passant de « l'individu ressource à l'individu sujet » pour une « gestion plus humaine des ressources » [DE GAULEJAC, 2005]. Avec la question déterminante de la confiance. Dans une approche systémique et « dialogique ", il s'agit de chercher à concilier ordre et désordre, qualitatif et quantitatif, individuel et collectif. C'est tout d'abord savoir reconnaître différentes logiques en tension, en remontant à la source des représentations: administrativo-économique de l'efficience, techniciste de l'outil parfait, souvent articulée à la logique marchande de grands groupes industriels, logiques professionnelles, correspondant souvent à des logiques de corps, logiques des patients, etc. Les logiques vont demeurer différentes, notamment entre secteurs privé et public, mais il s'agira d'essayer d'en limiter les tensions, en rendant les approches davantage collectives. Les moments d'échanges et de convivialité (notamment les formations) sont essentiels pour construire la confiance et la coopération entre tous les acteurs du réseau de santé. Les réunions le sont aussi pour favoriser la convergence des représentations entre acteurs, financeurs et évaluateurs pour essayer de limiter les conséquences des tensions entre coûts, normes et valeurs, devenues centrales dans le système de santé français et qui conditionnent largement son évolution et pour aider à construire des représentations partagées et une identité collective. Toujours dans une approche " dialogique », il s'agit aussi de dépasser les tensions entre temporalités (du court et du long terme): concilier l'urgence (à relier à l'approche administrativo-économique de l'efficience) et le durable (qualité des soins, privilégiée par les logiques professionnelles et par celle des patients).

Nous proposonsderaisonnerensituations d'informationetdecommunication (intérêt des différents cadres d'interprétation de la sémiotique situationnelle). Dans cette perspective, les réseaux de santé peuvent constituer des espaces de construction de nouvelles approches de l'évaluation, non seulement conçue, mais vécue comme démarche d'amélioration. L'intelligence collective des réseaux est avant tout l'intelligence du lien. Cette approche correspond aussi à une évolution vers une forme de coproduction de connaissances, voire de services, intégrant les savoirs de tous les partenaires, y compris le savoir expérientiel des patients et de leurs familles. La construction de nouveaux territoires de pratiques peut aussi relever d'une démarche d'intelligence territoriale de proximité.

Dans une logique objectifs/résultats, évaluer un réseau de santé, c'est surtout juger de la qualité de la coordination et, ce qui est plus difficile, des coopérations développées. Un des enjeux est celui de la traçabilité du parcours de soins des patients. Toute rupture est considérée comme un coût évitable 
(non qualité). Le réseau gérontologique étudié a ainsi fait des ruptures de parcours de soins évitées (recours aux urgences et réhospitalisations) un de ses objectifs prioritaires (estimation des coûts évités) et de l'évaluation de sa valeur ajoutée, avec son intégration dans le contexte local, l'écoute et la satisfaction des patients et de tous ses partenaires.

Le suivi du parcours de soins du patient et le renseignement d'indicateurs pour l'évaluation supposent la collecte de données fiables et validées. Il s'agit aussi de prendre en compte toute la spécificité des données de santé avec l'encadrement juridique de leur utilisation: responsabilité et secret médical. L'évaluation du fonctionnement des réseaux de santé est largement celle de leur système d'information, considéré comme révélateur de leur fonctionnement, de ses atouts comme de ses dysfonctionnements. Dans notre approche du réseau gérontologique évalué, nous l'avons abordé de manière très large, comme système d'information et de communication, incluant des dispositifs socio-techniques (outils informatiques: systèmes d'information ou dossiers informatisés patients, s'intégrant progressivement dans des plateformes de services), et prenant aussi en compte l'essentiel des interactions humaines.

\section{Conclusion}

Les réseaux de santé constituent un terrain d'observation privilégié pour analyser l'ambivalence de toute démarche d'évaluation (outil d'aide au développement mais aussi de contrôle). Dans une approche relevant de l'intelligence de la complexité, nous avons proposé une approche SIC de leur évaluation (privilégiant le sens, le lien, le savoir et l'action) pour essayer de limiter les tensions entre différentes logiques à l'œuvre dans les réseaux de santé et, plus largement, dans tout notre système de santé, en insistant sur la convergence des représentations et la prise en compte des contextes, pour en dépasser les cloisonnements. Nous nous situons dans une approche de l'évaluation "équitable » [DEJOURS, 2010] ou "dynamique » [DE GAULEJAC, 2005], comme amélioration, aide au développement, en intégrant le plus possible la parole des acteurs pour rendre visible leur travail coopératif, en privilégiant la relation et le sens, avec toute l'importance de la dimension pédagogique (formation), de la reconnaissance et de la confiance. Il s'agit d'estimer la dynamique des coopérations et non la seule conformité à des normes.

Organisations d'interface «hologrammatiques » (le tout est dans la partie et la partie dans le tout) des grands changements en cours dans le système de santé français, les réseaux sont désormais en concurrence avec d'autres organisations d'interface. Remis en question depuis 2006, ils constituent néanmoins des formes organisationnelles permettant la construction de la coopération et de la confiance entre tous leurs acteurs, en valorisant leur expérience (organisation apprenante). Ils sont aussi des espaces projets pour l'expérimentation de nouvelles approches de l'évaluation - amélioration, en 
essayant d'intégrer les représentations de tous les acteurs et partenaires pour dépasser les logiques en tension.

Cette approche del'évaluation-amélioration, centrée surl'interdisciplinarité des SIC, correspond à l'émergence de territoires de pratiques coopératives, relevant d'une démarche d'intelligence sociétale et territoriale. Dans une perspective d' " économie de la relation » [D'ALMEIDA, 2001], cette approche de l'évaluation peut s'intégrer dans la perspective souhaitée par P. Rosanvallon [ROSANVALLON, 1998] pour envisager de nouvelles pistes permettant de « repenser l'État providence » et la solidarité.

\section{BIBLIOGRAPHIE}

BARRÉ S., ÉVIN C., FOURÉ P.-Y., HOUDART L., LAROSE D., POUTOUT G., PTAKHINE E., Traité pratique des réseaux de santé, Paris : Berger-Levrault, 2005, $456 \mathrm{p}$.

BOUGNOUX D. Introduction aux sciences de la communication, Paris : La Découverte, 2001, 126 p.

BOUSSARD V. Sociologie de la gestion. Les faiseurs de performance. Paris : Belin, 2008.

D'ALMEIDA N. Les promesses de la communication. Paris: PUF, 2001, rééd., 2007, $264 \mathrm{p}$.

DE GAULEJAC V. La société malade de la gestion. Idéologie gestionnaire, pouvoir managérial et harcèlement social. Paris: 2005, nouvelle éd., Points-Seuil, mars 2009, 353 p.

DEJOURS C. L'évaluation du travail à l'épreuve du réel. Critique des fondements de l'évaluation, Paris: INRA Éditions, 2010, 82 p.

GRAMACCIA G., Les actes de langage dans les organisations, Paris, L'Harmattan, 2001, 287 p.

HEINICH N. Pour une neutralité engagée. Questions de communication, actes III, 2006, pp. $83-96$.

LE MOËNNE C. La communication organisationnelle à l'heure de la dislocation spatiotemporelle des entreprises. La mutation des formes organisationnelles, Sciences de la Société, Toulouse, LERASS, Presses Universitaires du Mirail, n 62, 2004, pp. 209 - 224.

LIEVRE P. Évaluer une action sociale. Rennes: Éditions ENSP, 2002, 115 p.

MINTZBERG H. Le Management. Voyage au centre des organisations. Paris: Ed. d'Organisation, 2001, $570 \mathrm{p}$.

MORIN E., LE MOIGNE J.-L. L'intelligence de la complexité. Paris: L'Harmattan, 2003, $332 \mathrm{p}$.

MUCCHIELLI A. Situation et Communication. Nice: Ed. Ovadia, 2010, 170 p.

ROSANVALLON P. La nouvelle question sociale. Repenser l'État-providence. Paris: Le Seuil, Points-Essais, 1998.

SCHWEYER F.-X., LEVASSEUR G., PAWLIKOWSKA T., Créer et piloter un réseau de santé, Rennes : Éditions ENSP, 2002, 112 p. 
THÉVENOT L. Un gouvernement par les normes. Pratiques et politiques des formats d'information. In : CONEIN B. et THEVENOT L., dir. Cognition et information en société. Paris : Éditions de l'EHESS, 1997, pp. $205-242$.

Résumé: Les cloisonnements du système de santé français, notamment entre la médecine de ville et le secteur hospitalier, sont considérés comme une des causes de ses dysfonctionnements. Le développement d'organisations d'interface et, en particulier, des réseaux de santé, a constitué une réponse possible, dans un contexte où le rôle de l'État providence est en pleine évolution. Leur expérimentation a rencontré la question de l'ambivalence de leur évaluation: aide au développement mais aussi contrôle (voire sanction).

Une approche Sciences de l'Information et de la Communication de l'évaluation des réseaux de santé peut permettre de faire converger les représentations des différents acteurs et partenaires (y compris patients) sur les problématiques du lien, du sens, du savoir et d'action, en s'appuyant sur l'analyse de situations d'information et de communication. Cherchant à concilier aspects qualitatifs et quantitatifs, coûts, normes et valeurs, comme temporalités de l'urgence et du durable pour surmonter des logiques en tension, cette approche positionne l'évaluation dans une perspective d'aide au développement des réseaux ou d'autres organisations d'interface en santé.

Mots clés : Réseaux de santé, évaluation, interface, développement, coopération.

\begin{abstract}
The partitions in the French Healthcare System are considered as one of the causes of its problems, especially "walls" between primary care and the hospital sector. The development of interface organizations, particularly Healthcare Networks, may be an answer to this problem in a context where the role of the Welfare State is changing. Their experiment met the issue of ambivalence of their evaluation: to help improvement or to control (even to sanction). An approach through Information and Communication Sciences of the evaluation of Healthcare Networks may allow the convergence of representations of different actors and partners (including patients) towards the issues of link, meaning, knowledge and action, based on the analysis of information and communication's situations. Trying to reconcile qualitative with quantitative aspects, costs, standards with values, such as temporality of urgency with sustainability to overcome opposed logics, this approach sets the evaluation as an help in the development of Networks or other Health Interfaces Organizations.
\end{abstract}

Key Words: Healthcare Networks, evaluation, interface, development, cooperation. 